\title{
Borderline personality disorder is associated with an increased risk of sexually transmitted infections: A nationwide population-based cohort study in Taiwan
}

\author{
Shih-Fen Chen \\ Center of Medical Genetics, Hualien Tzu Chi Hospital, Buddhist Tzu Chi Medical Foundation, Hualien, Taiwan
}

\section{Background}

Several features of borderline personality disorder (BPD) are likely to be associated with sexual health problems, such as unstable attachment, unstable sexual identity, and sexual impulsivity. Since Taiwan's society is hidden to discuss the issue of sex, sexual health needs, including screening and prevention of sexually transmitted infections (STI), are often neglected in this population.

\section{Objective}

The study aims to determine whether BPD patients are associated with an increased risk of subsequent STI in Taiwan.

\section{Methods}

An overall of 669 BPD patients and 2676 controls matched by gender and age were enrolled between 2000 and 2012 and followed until the end of 2013 using Taiwan's National Health Insurance Research Database. During the follow-up period, participants who developed STI (human immunodeficiency virus, syphilis, genital warts, gonorrhoea, chlamydia, and trichomoniasis) were identified. Cox regression analysis was used to calculate the hazard ratio $(\mathrm{HR})$ with $95 \%$ confidence interval $(95 \% \mathrm{Cl})$ of the STI incidence rate between BPD patients and unaffected controls.

\section{Results}

BPD patients were predisposed to developing STI (hazard ratio $(\mathrm{HR}): 4.17,95 \%$ confidence interval $(95 \%$ $\mathrm{Cl})$ : 1.62-10.8) after adjusting for demographic data and psychiatric comorbidities. The stratification analysis revealed a similar risk trend with BPD and subsequent STI in each gender and age group and were significant in the subgroups of male (HR: $11.3,95 \% \mathrm{Cl}: 2.97-42.7$ ), and those aged 18 to 34 years (HR: $4.85,95 \% \mathrm{Cl}: 1.71$ 13.7). Also, the comorbidity stratification analysis revealed that, when the effect of comorbidities was excluded, pure BPD patients significantly exhibited the risk association for subsequent STI after adjusting for all variables (HR: 4.24, $95 \% \mathrm{Cl}: 1.25-14.4)$.

\section{Conclusions}

Given the greater potential for BPD patients associated with an increased risk of STI, there should be direct implications for the development of targeted prevention interventions in Taiwan's mental health clinics.

Keywords: borderline personality disorder, sexually transmitted infections, human immunodeficiency virus, sexual health, comorbidity, cohortt

References:

1. Chen EY, Brown MZ, Lo TT, Linehan MM. Sexually transmitted disease rates and high-risk sexual behaviors in borderline personality disorder versus borderline personality disorder with substance use disorder. J Nerv Ment Dis 2007:195:125-9.

2. Harned MS, Pantalone DW, Ward-Ciesielski EF, Lynch TR, Linehan MM. The prevalence and correlates of sexual risk behaviors and sexually transmitted infections in outpatients with borderline personality disorder. J Nerv Ment Dis $2011 \cdot 199 \cdot 832-8$.
Table 1: Demographic characteristics of patients with BPD and non-BPD controls

\begin{tabular}{|c|c|c|c|c|c|}
\hline \multirow{3}{*}{ Variable } & \multirow{2}{*}{\multicolumn{2}{|c|}{$\begin{array}{c}\text { BPD } \\
n=669\end{array}$}} & \multirow{2}{*}{\multicolumn{2}{|c|}{$\begin{array}{l}\text { Control } \\
n=2676\end{array}$}} & \multirow{3}{*}{ P-value } \\
\hline & & & & & \\
\hline & $\mathrm{n}$ & $\%$ & $\mathbf{n}$ & $\%$ & \\
\hline Gender & & & & & 0.99 \\
\hline Female & 406 & 60.7 & 1624 & 60.7 & \\
\hline Male & 263 & 39.3 & 1052 & 39.3 & \\
\hline Age at baseline, years ${ }^{a}$ & & & & & 0.99 \\
\hline $18-34$ & 455 & 68.0 & 1820 & 68.0 & \\
\hline $35-50$ & 164 & 24.5 & 656 & 24.5 & \\
\hline$>50$ & 50 & 7.5 & 200 & 7.5 & \\
\hline Median $(\mathrm{IQR})^{\mathrm{b}}$ & \multicolumn{2}{|c|}{$29.2(23.0-37.8)$} & \multicolumn{2}{|c|}{$29.3(22.0-38.5)$} & 0.25 \\
\hline \multicolumn{6}{|l|}{ Comorbidity $^{\mathrm{a}}$} \\
\hline Depressive disorder & 521 & 77.9 & 90 & 3.4 & $<.01$ \\
\hline Bipolar disorder & 169 & 25.3 & 13 & 0.5 & $<.01$ \\
\hline Anxiety disorder & 319 & 47.7 & 189 & 7.1 & $<.01$ \\
\hline Substance use disorder & 189 & 28.3 & 44 & 1.6 & $<.01$ \\
\hline Follow-up period, years (IQR) & \multicolumn{2}{|c|}{$6.2(3.0-9.3)$} & \multicolumn{2}{|c|}{$7.0(4.0-9.9)$} & $<.01$ \\
\hline
\end{tabular}

: chi-square test; : Wilcoxon's rank-sum test.
BPD: borderline personality disorder; IQR: interquartile range

Table 2: Cox regression analyses of overall STI risk among cohorts of BPD and control, then stratified by gender, age, and comorbidity.

\begin{tabular}{|c|c|c|c|c|c|c|c|c|}
\hline \multirow[b]{2}{*}{ Variable } & \multicolumn{3}{|c|}{ BPD } & \multicolumn{3}{|c|}{ Control } & \multirow{2}{*}{$\begin{array}{c}\text { Crude }^{b} \\
\text { HR } \\
(95 \% \mathrm{Cl})\end{array}$} & \multirow{2}{*}{$\begin{array}{c}\text { Adjusted }^{c} \\
\text { HR } \\
(95 \% \mathrm{Cl})\end{array}$} \\
\hline & STI & $\begin{array}{l}\text { Person } \\
\text { years }\end{array}$ & $\mathbf{I R}^{\mathbf{a}}$ & STI & $\begin{array}{c}\text { Person } \\
\text { years }\end{array}$ & $\mathbf{I R}^{\mathbf{a}}$ & & \\
\hline Total & 21 & 4281 & 4.91 & 26 & 18690 & 1.39 & $\begin{array}{c}3.52 \\
(1.98-6.25)^{\star}\end{array}$ & $\begin{array}{c}4.17 \\
(1.62-10.8)^{*}\end{array}$ \\
\hline $\begin{array}{l}\text { Sensitivity } \\
\text { analysis }^{d}\end{array}$ & 20 & 4278 & 4.67 & 26 & 18690 & 1.39 & $\begin{array}{c}3.36 \\
(1.87-6.01)^{*}\end{array}$ & $\begin{array}{c}3.41 \\
(1.25-9.32)\end{array}$ \\
\hline \multicolumn{9}{|l|}{ Gender } \\
\hline Female & 14 & 2577 & 5.43 & 21 & 11257 & 1.87 & $\begin{array}{c}2.92 \\
(1.49-5.75)^{\star}\end{array}$ & $\begin{array}{c}1.71 \\
(0.44-6.69)\end{array}$ \\
\hline Male & 7 & 1704 & 4.11 & 5 & 7434 & 0.67 & $\begin{array}{c}6.04 \\
(1.92-19.1)^{\star}\end{array}$ & $\begin{array}{c}11.3 \\
(2.97-42.7)^{\star}\end{array}$ \\
\hline \multicolumn{9}{|l|}{$\begin{array}{c}\text { Age group, } \\
\text { year }\end{array}$} \\
\hline $18-34$ & 15 & 3021 & 4.97 & 19 & 12984 & 1.46 & $\begin{array}{c}3.39 \\
(1.72-6.68)^{\star}\end{array}$ & $\begin{array}{c}4.85 \\
(1.71-13.7)^{\star}\end{array}$ \\
\hline $35-50$ & 6 & 980 & 6.12 & 5 & 4509 & 1.11 & $\begin{array}{c}5.36 \\
(1.64-17.6)^{\star}\end{array}$ & $\begin{array}{c}2.35 \\
(0.22-25.8)\end{array}$ \\
\hline$>50$ & 0 & 279 & 0.00 & 2 & 1198 & 1.67 & - & - \\
\hline \multicolumn{9}{|c|}{ Comorbidity } \\
\hline Any $^{\mathrm{e}}$ & 18 & 3655 & 4.93 & 4 & 1660 & 2.41 & $\begin{array}{c}2.13 \\
(0.72-6.28)\end{array}$ & $\begin{array}{c}1.97 \\
(0.66-5.91)\end{array}$ \\
\hline None & 3 & 626 & 4.79 & 22 & 17030 & 1.29 & $\begin{array}{c}3.57 \\
(1.07-11.9)^{*}\end{array}$ & $\begin{array}{c}4.24 \\
(1.25-14.4)^{\star}\end{array}$ \\
\hline
\end{tabular}

: incidence rates, per 1000 person-years; ${ }^{b}$ : relative hazard ratio; : $:$ mutually adjusted for BPD, gender, age, and comorbidities in Cox regression analyses; ': eliminate STI incidents with an interval less than 6 months 作 anxiety disorder, and substance use disorder; ${ }^{*} p<0.05$

STI: sexually transmitted infections; BPD: borderline personality disorder; IR: incidence rates; HR: hazard ratio; Cl: confidence interval. 